\title{
Le principe de libre administration des collectivités territoriales dans la jurisprudence du Conseil d'État
}

The principle of the free administration of local authorities in the case law of the Council of State

\section{Emmanuelle Borner-Kaydel}

\section{OpenEdition \\ Journals}

Édition électronique

URL : http://journals.openedition.org/add/328

DOI : $10.4000 /$ add.328

ISSN : 2606-1988

Éditeur

Presses universitaires de Rouen et du Havre

Édition imprimée

Date de publication : 1 juin 2016

Pagination : 09-32

ISBN : 979-10-240-0599-7

ISSN : 1955-0855

\section{Référence électronique}

Emmanuelle Borner-Kaydel, « Le principe de libre administration des collectivités territoriales dans la jurisprudence du Conseil d'État », Les Annales de droit [En ligne], 10 | 2016, mis en ligne le 08 janvier 2018, consulté le 30 avril 2019. URL : http://journals.openedition.org/add/328 ; DOI : 10.4000/add.328 


\title{
Le principe de libre administration des collectivités territoriales dans la jurisprudence du Conseil d'État
}

\author{
Emmanuelle BORNER-KAYDEL
}

«On peut gouverner de loin, on administre bien que de près ${ }^{1}$.» L'idée de ne pas concentrer l'intégralité de l'administration de la France entre les seules mains du pouvoir étatique s'inscrit dans un contexte historique de longue haleine. Toutefois, les grandes réformes ayant mis en place de manière effective cette répartition des pouvoirs entre l'autorité étatique et les collectivités territoriales ne se sont réalisées que dans le dernier quart du vingtième siècle, et de nouveaux changements sont encore en attente.

L'actuel paysage territorial de la France, avec ses découpages géographiques et politiques multiples, n'en demeure pas moins respectueux du principe d'unité et d'indivisibilité de la République ${ }^{2}$. En effet, la France étant à la fois un des premiers États-nations mais aussi un des derniers encore actuels ${ }^{3}$, il importait de ne pas sacrifier le caractère unitaire de la République au profit d'une gestion décentralisée de la France dans laquelle le pouvoir étatique perdrait toute compétence. Dès lors, les différentes étapes de la construction de ce paysage n'ont pas eu pour conséquence d'aboutir à une absorption d'un pouvoir par l'autre, mais bien plutôt à une complémentarité entre eux. Si la Constitution de 1958 consacre dès son article premier le principe de l'indivisibilité de la République, ce même article ajoute plus loin que «son organisation est décentralisée ${ }^{4}$ ", mettant ainsi sur un plan d'égalité l'importance de la forme de l'État et de la manière de l'administrer. Les grandes

1. D. $\mathrm{n}^{\mathrm{0}} 3855,25$ mars 1852 , décentralisation administrative.

2. Art. 1 de la Constitution du 4 octobre $1958:$ :La France est une République indivisible $[\ldots] »$

3. Olivier Gohin, «Les formes de l'État français depuis 1958 », Politeia, $\mathrm{n}^{0}$ 12, 2007, p. 215.

4. Art. 1 de la Constitution du 4 octobre 1958. 
réformes portant sur la décentralisation ${ }^{5}$ répondent à l'affirmation de François Mitterrand selon laquelle : «La France a eu besoin d'un pouvoir fort et centralisé pour se faire. Elle a aujourd'hui besoin d'un pouvoir décentralisé pour ne pas se défaire ${ }^{6}$ ». Le terme "décentralisation " est symboliquement consacré ${ }^{7}$ à l'occasion de la loi de 1988 relative à l'amélioration de la décentralisation ${ }^{8}$. Puis, en $1992^{9}$, le législateur confie l'administration territoriale de la République aux collectivités territoriales. Enfin, marquant ce qui a été appelé «la décentralisation, Acte II ", la révision constitutionnelle de $2003{ }^{10}$ permet à l'administration décentralisée de la France de trouver une assise constitutionnelle et d'ainsi renforcer sa protection.

À l'origine, le principe était celui de la libre administration des collectivités territoriales, et non celui de la décentralisation ${ }^{11}$. La Constitution de 1946 précisait que « les collectivités territoriales s’administrent librement $^{12}$ ». Et l'article 72 de la Constitution de 1958 reprend les mêmes termes pour préciser que les collectivités territoriales s'administrent librement. Aujourd'hui, si le terme "décentralisation » est bien ancré dans le paysage législatif et constitutionnel français, c'est au principe de libre administration des collectivités territoriales que continue de se référer la jurisprudence ${ }^{13}$, tant constitutionnelle qu'administrative. Et, si la loi de 1982 a posé les principes fondateurs de la décentralisation tandis que la révision de 2003 en a consolidé l'assise tout en ajoutant quelques principes novateurs ${ }^{14}$, il faut toutefois admettre que cette loi

5. L. $n^{\circ} 82-213,2$ mars 1982 relative aux droits et libertés des communes, des départements et des régions; L. $\mathrm{n}^{0} 83-8,7$ janv. 1983 relative à la répartition de compétences entre les communes, les départements, les régions et l'État, dite «Loi Defferre »; L. $\mathrm{n}^{\circ}$ 84-53, 26 janv. 1984 portant dispositions statutaires relatives à la fonction publique territoriale.

6. François Mitterrand, Conseil des Ministres du 15 juillet 1981, cité dans Michel Verpeaux, "1982: de quoi la loi du 2 mars est-elle la cause?", AJDA 2012, $\mathrm{n}^{\circ}$ 14, p. 743.

7. Michel Verpeaux, «1982: de quoi la loi du 2 mars est-elle la cause? ?, art. cité, p. 744.

8. L. $\mathrm{n}^{\mathrm{0}} 88-13,5$ janv. 1988 relative à l'amélioration de la décentralisation.

9. L. ${ }^{0}$ 92-125, 6 févr. 1992 relative à l'administration territoriale de la République.

10. L. const. $\mathrm{n}^{\mathrm{O}} 2003-276,28$ mars 2003 relative à l'organisation décentralisée de la République.

11. Michel Verpeaux, «1982: de quoi la loi du 2 mars est-elle la cause ?», art. cité, p. 743.

12. Art. 87 de la Constitution du 27 octobre 1946.

13. Art. 72, al. 3 de la Constitution du 4 octobre 1958.

14. Évelise Plenet, «La réforme des collectivités territoriales: quand la Constitution s'en mêle», Droit et société, $\mathrm{n}^{0}$ 81, mai 2012, p. 386. 
constitutionnelle a dans le même temps resserré l'évolution des collectivités territoriales dans un « carcan beaucoup plus précis ${ }^{15}$ ".

S'agissant des collectivités territoriales, la Constitution précise qu'elles sont les communes, les départements, les régions, mais aussi les collectivités à statut particulier ainsi que les collectivités d'outre-mer ${ }^{16}$. Une collectivité territoriale peut se définir comme étant une «entité de droit public correspondant à des groupements humains géographiquement localisés sur une portion déterminée du territoire national, [à laquelle] l'État a conféré la personnalité juridique et le pouvoir de s'administrer par des autorités élues ${ }^{17}$ ».

La question que soulève l'étude du principe de libre administration des collectivités territoriales est celui de l'effectivité de sa protection. En effet, ce principe ne connaît aucune définition juridique précise et déterminée. Rechercher si le droit pour les collectivités de s'administrer librement est suffisamment protégé amène à s'interroger sur le contenu du principe de libre administration. L'étude de la jurisprudence du Conseil d'État sur ce point se révèle très opportune. Il est en effet possible d'y déceler des éléments de définition, ainsi que des principes de mise en œuvre. La protection d'un tel principe semble également dépendre du degré de contrôle qui est exercé, en recherchant si un équilibre est respecté entre la latitude qui échoit aux collectivités et la légalité des actes qu'elles peuvent édicter. Ainsi, se pose la question de savoir dans quelle mesure les actes pris par les collectivités territoriales entrent dans le champ de leur libre administration et sont rendus effectifs. Il importe pour cela de s'interroger sur la manière dont ces actes demeurent encadrés, mais aussi de rechercher l'étendue de l'autonomie des collectivités dans leur pouvoir de décision. Il conviendra dès lors de s'intéresser dans un premier temps au champ d'application du principe de libre administration des collectivités territoriales (1) et, dans un second temps, à l'effectivité de ce principe (2).

15. Didier Maus, «La réformes des collectivités locales: un casse-tête constitutionnel?», Revue politique et parlementaire, $\mathrm{n}^{\circ}$ 1053, 2009, p. 82.

16. Art. 72, al. 1 de la Constitution de 1958.

17. Raymond Guillien, Jean Vincent, Serge Guinchard et Gabriel Montagnier, Lexique des termes juridiques, Paris, Dalloz, 13 éd., 2001, p. 108-109. 


\section{Le champ d'application du principe de libre administration des collectivités territoriales}

Le Conseil d'État est régulièrement saisi d'une question entourant le principe de libre administration des collectivités territoriales. La question principale demeure celle de la reconnaissance d'une atteinte à ce principe, le plus souvent par un acte pris par exemple par une commune ou un préfet. Or, le juge administratif n'admet que rarement l'atteinte au principe de la libre administration des collectivités. En outre, il n'a pas encore livré de véritable définition précise de ce principe. Dès lors, peut se poser la question de savoir si «la définition a minima de la liberté d'administration des collectivités ${ }^{18}$ " par le juge administratif suffit à garantir une protection effective à ce principe. L'effectivité de la libre administration des collectivités terrioriales se renforce chaque fois que le Conseil d'État apporte de nouveaux éléments de définition de ce principe (1.1), et se mesure en fonction du contrôle exercé sur les actes pris par les collectivités territoriales en vertu de leur pouvoir de libre administration (1.2).

\subsection{Les éléments de définition de la libre administration des collectivités territoriales}

Le Conseil d'État a érigé le principe de libre administration des collectivités territoriales au rang de liberté fondamentale à l'occasion de l'ordonnance de référé Commune de Beaulieu-sur-Mer contre ministre de l'Intérieur, en $2002^{19}$, position réitérée par une ordonnance de $2006^{20}$. Mais il faut entendre ici par "liberté fondamentale» le sens que lui donne l'article L. 521-2 du code de justice administrative, lequel dispose : «[...] le juge des référés peut ordonner toutes mesures nécessaires à la sauvegarde d'une liberté fondamentale à laquelle une personne morale de droit public ou un organisme de droit privé chargé de la gestion d'un service public aurait porté, dans l'exercice d'un de ses pouvoirs, une atteinte grave et manifestement illégale ${ }^{21}$.

Cette élévation au rang de liberté fondamentale au sens du code de justice administrative, est confirmée par une jurisprudence constante du

18. Nelly Ferreira, «La convocation des conseillers municipaux relève des rapports internes», AJDA 2013, $n^{0} 13$, p. 741.

19. CE, 24 janv. 2002, $\mathrm{n}^{\circ}$ 242128, Commune de Beaulieu-sur-Mer contre ministre de l'Intérieur.

20. CE, $1^{\mathrm{er}}$ mars 2006, $\mathrm{n}^{\mathrm{0}}$ 290417, Ministre délégué aux collectivités territoriales.

21. Art. L. 521-2 du code de justice administrative, créé par la loi $n^{0}$ 2000-597 du 30 juin 2000. 
Conseil d'État. Ainsi, par exemple, l'arrêt Commune de Venelles ${ }^{22}$ précise que «le principe de libre administration des collectivités territoriales, énoncé par l'article 72 de la Constitution, est au nombre des libertés fondamentales auxquelles le législateur a entendu accorder une protection juridictionnelle particulière ${ }^{23} »$. Cependant, cette consécration n'en demeure pas moins "marquée par une certaine imprécision ${ }^{24}$ ». La jurisprudence du Conseil d'État en la matière y remédie quelque peu en établissant le contenu du principe de la liberté d'administration des collectivités, esquissant ainsi une définition à la fois positive et négative de ce principe, expliquant ce qui le concerne et ce qui ne le concerne pas. Ainsi, est une composante de la libre administration des collectivités territoriales le principe du refus par une commune d'être incluse d'office dans une communauté de communes ${ }^{25}$. Dès lors, les collectivités territoriales, pour pouvoir s'administrer librement, doivent pouvoir choisir leur inclusion au sein d'un groupe de collectivités, et non pas se voir imposer celle-ci contre leur gré. Dans le même sens, le Conseil d'État estime qu'il y a atteinte au principe de libre administration des collectivités lorsque, à l'occasion d'un transfert de compétences résultant de l'extension-transformation d'une communauté de communes en communauté d'agglomération, un établissement public de coopération intercommunale (ci-après, EPCI) a commencé à exercer ses nouvelles compétences alors que l'arrêté signé n'avait pas encore de date d'effet ${ }^{26}$. En revanche, le juge administratif ne considère pas qu'il y ait atteinte au principe de libre administration des collectivités territoriales lorsque le poste de président du syndicat mixte d'un parc naturel régional est vacant, même suite à un refus de la part de la région de lui en céder la présidence ${ }^{27}$. Le fait qu'une commission syndicale ne soit pas constituée n'est pas non plus reconnu comme une atteinte à ce principe ${ }^{28}$. Enfin, si l'arrêt Commune de Vennelles précité confirme la nature juridique de la libre administration des collectivités territoriales, elle n'en restreint

22. CE, 18 janv. 2001, $\mathrm{n}^{\mathrm{o}}$ 229247, Commune de Vennelles contre Morbelli.

23. Ibid., considérant 3.

24. Louis Favoreu et André Roux, «La libre administration des collectivités territoriales est-elle une liberté fondamentale?», Cah. Cons. Const. 2002, $\mathrm{n}^{0} 12$.

25. Marie-Christine Rouault, «Libre administration des collectivités et communautés de communes ", Revue Lamy des collectivités territoriales, mai 2006, $\mathrm{n}^{\circ} 13, \mathrm{p} .35$.

26. CE, 12 juin 2002, $\mathrm{n}^{\mathrm{O}}$ 246618, Commune de Fauillet, Montpouillan et Ste-Marthe contre communauté d'agglomération Val-de-Garonne.

27. CE, 8 mars 2002, $\mathrm{n}^{\circ}$ 238679, Région Languedoc-Roussillon.

28. CE, 28 avr. 2003, $\mathrm{n}^{\circ}$ 235111, Fédération nationale des ayants-droits de section de commune. 
pas moins le champ d'application en excluant de ce principe les rapports internes des collectivités territoriales ${ }^{29}$. En l'espèce, le Conseil d'État estime que le refus du maire de la commune de convoquer le conseil municipal, en dépit des demandes qui lui avaient été faites en ce sens, "ne concerne que les rapports internes au sein de la commune et ne peut, par suite, être regardé comme méconnaissant ${ }^{30}$ " le principe de libre administration des collectivités territoriales.

Les rapports internes des collectivités territoriales semblent dès lors être à la fois «la limite et le critère ${ }^{31}$ » de leur libre administration. Il semble ressortir de la jurisprudence du Conseil d'État qu'à partir du moment où la question touche aux rapports internes des collectivités, aucune atteinte au principe de leur libre administration ne peut être relevée. Ces rapports internes deviennent ainsi la délimitation matérielle du principe de libre administration des collectivités territoriales en ce sens que "tout ce qui ne relève pas des rapports internes peut se rattacher à cette liberté ${ }^{32}$ ». Ces rapports internes concernent aussi bien le refus d'un maire de communiquer à un élu local notamment la liste électorale ${ }^{33}$, que le fonctionnement irrégulier d'un $\mathrm{EPCI}^{34}$. En s'intéressant à la jurisprudence des tribunaux administratifs, il est loisible de s'apercevoir qu'ils statuent dans le même sens que le Conseil d'État, et admettent au nombre des rapports internes la décision prise par une assemblée locale de révoquer les adjoints de leurs fonctions ${ }^{35}$, ainsi que le refus du président de l'assemblée d'un EPCI de donner la parole à un élu lors d'une séance ${ }^{36}$.

Le principe de libre administration des collectivités territoriales, s'il ne bénéficie pas encore d'une définition précise, présente un contenu assez étoffé et clairement expliqué par le juge administratif. Sa valeur constitutionnelle liée à son inscription au sein de l'article 72 de la Constitution ainsi que sa nature juridique de liberté fondamentale, posée

29. Marie-Christine Rouault, «Libre administration des collectivités et communautés de communes", art. cité, p. 36.

30. CE, 18 janv. 2001, $\mathrm{n}^{\circ}$ 229247, Commune de Vennelles et Morbelli, considérant 3.

31. Nelly Ferreira, "La convocation des conseillers municipaux relève des rapports internes", art. cité, p. 742.

32. Ibid.

33. CE, 7 fév. 2001, $\mathrm{n}^{0}$ 229921, Commune de Pointe-à-Pitre.

34. CE, 8 mars 2002, Région Languedoc-Roussillon.

35. TA Melun, 31 déc. 2004, req. $\mathrm{n}^{\mathrm{o}}$ 046915, L. Koessler et $M^{m e}$ D. Bourdet contre commune de Villeneuve-le-Roi.

36. TA Strasbourg, 2 mars 2011, req. $n^{\circ} 1100962$. 
par le Conseil d'État et confirmée par le tribunal des conflits ${ }^{37}$ s'appuyant sur la théorie de la voie de fait ${ }^{38}$, laissent supposer une ouverture au nouveau mécanisme de la question prioritaire de constitutionnalité. L'instauration de ce mécanisme vient ainsi enrichir le contrôle des actes des collectivités territoriales, opéré jusqu'alors par le préfet.

\subsection{Le contrôle accru des actes des collectivités territoriales}

La mise en place de la question prioritaire de constitutionnalité (ciaprès, QPC) ouvre un nouvel horizon aux collectivités territoriales en conflit par rapport à leur droit de s'administrer librement. En effet, si les collectivités sont libres de s'administrer par elles-mêmes, cela ne doit pas se comprendre comme un droit de faire n'importe quoi. Force est de constater que certains actes pris par une commune sont parfois entachés d'irrégularité. Le contrôle de légalité opéré par le préfet ne récolte pas l'ensemble des suffrages et semble décrié en ce qu'il serait insuffisant ${ }^{39}$. La faiblesse de ce contrôle s'observe en particulier lorsque les collectivités territoriales, dont les actes n'ont pas été contrôlés, sont attaquées devant le juge administratif par la voie du recours pour excès de pouvoir par les particuliers ${ }^{40}$. La jurisprudence du Conseil d'État en la matière est très claire. Il précise en premier lieu que le pouvoir du préfet de déférer un acte d'une collectivité territoriale est discrétionnaire ${ }^{41}$ et qu'il peut se désister en cours d'instance, à tout moment, sans avoir à faire contrôler les motifs de son désistement ${ }^{42}$. Par suite, le juge administratif ajoute qu'en cas d'abstention du préfet, celle-ci n'est de nature à engager la responsabilité de l'État qu'à la condition que la carence ainsi observée résulte d'une faute lourde ${ }^{43}$. Enfin, le Conseil d'État précise que la faute lourde est constituée en cas d'abstention prolongée par le préfet de

37. TC, 19 nov. 2007, req. $\mathrm{n}^{\circ}$ 3653, Maire de Limeil-Brévannes contre préfet de Valde-Marne.

38. Charles-André Dubreuil, "Libre administration des collectivités territoriales et liberté fondamentale », JCP G 2012, $n^{\circ}$ 46, p. 2048.

39. Marguerite Canedo-Paris, «Le principe de libre administration et la règle "nemo auditur" dans le contentieux du déféré préfectoral », Les Petites Affiches ( LPA»), $\mathrm{n}^{\mathrm{o}}$ 104, 26 mai 2010, p. 12.

40. Bernard Poujade, «Filtrer le moustique et laisser passer le chameau : le contrôle de légalité aujourd'hui? », BJCL, $\mathrm{n}^{\circ}$ 11/13, 2013, p. 721.

41. CE, 25 janv. 1991, $\mathrm{n}^{\circ} 80969$, Brasseur.

42. CE, 16 juin 1989, $\mathrm{n}^{\circ}$ 103661, Préfet des Bouches-du-Rhône contre commune de Belcodène.

43. $\mathrm{CE}, 21$ juin 2000, $\mathrm{n}^{\mathrm{0}}$ 202058, Ministre de l'équipement contre commune de Roquebrune-Cap-Martin. 
déférer des actes importants dont l'illégalité était manifeste et connue ${ }^{44}$, et qu'en cas d'annulation d'un acte par le préfet, celle-ci ne présente pas un caractère rétroactif ${ }^{45}$. La discrétion du pouvoir du préfet, sa propension à ne pas déférer les actes pris par les collectivités territoriales dans le cadre de leur libre administration, et l'exigence d'une faute lourde, aboutissent à un bilan partagé quant à l'opportunité et l'efficacité du contrôle de légalité des actes des collectivités par le préfet, contrôle que la doctrine qualifie parfois de "contrôle à éclipses et techniquement incertain $^{46}$ ».

C'est la raison pour laquelle le contrôle opéré par la QPC est porteur d'un espoir nouveau pour les administrés ou pour les communes qui souhaitent protéger au mieux leur droit de s'administrer librement. Mais le recours à la question prioritaire de constitutionnalité ne saurait être considéré comme acquis s'agissant du contrôle des actes des collectivités, et la première question à se poser est celle de savoir si la libre administration des collectivités territoriales peut fonder un recours devant le Conseil constitutionnel. La réponse apportée s'est faite en deux temps. Dans un premier temps, le juge constitutionnel, saisi par le Conseil d'État, ne se prononce qu'implicitement ${ }^{47}$ en faveur d'une telle possibilité, à l'occasion de la première décision QPC en matière de collectivités territoriales ${ }^{48}$, en 2010. En effet, en jugeant que la décision de procéder à une fusion de communes ne porte pas atteinte au principe de leur libre administration ${ }^{49}$, le Conseil constitutionnel montre son acceptation de ce principe en tant que fondement d'une question prioritaire de constitutionnalité. En l'espèce, deux communes souhaitent fusionner et transmettent pour ce faire les délibérations concordantes de leurs conseils municipaux au préfet, qui soumet cette décision à l'avis des habitants des communes concernées ${ }^{50}$. Le résultat du référendum

44. CE, 6 oct. 2000, $\mathrm{n}^{\circ}$ 205959, Ministre de l'Intérieur contre commune de St-Florent et autres.

45. CE, 4 juil. 2012, $\mathrm{n}^{\circ}$ 352417, Communauté d'agglomération de Chartres Métropole.

46. Bernard Poujade, "Filtrer le moustique et laisser passer le chameau...», art. cité, p. 723.

47. Armelle Trepoz Bruant, «Libre administration des collectivités territoriales et QPC: entre espoir et amertume ", Droit administratif, $\mathrm{n}^{\circ}$ 12, décembre 2012, p. 12.

48. Marie-Christine Rouault, «La procédure de consultation des électeurs en matière de fusion de communes est-elle inconstitutionnelle?», Revue Lamy des collectivités territoriales, $\mathrm{n}^{\circ} 60$, septembre 2010, p. 45 .

49. Cons. Const., déc. 2 juil. 2010, $\mathrm{n}^{\circ}$ 2010-12 QPC, Commune de Dunkerque, cons. 4.

50. Michel Verpeaux, "Question prioritaire de constitutionnalité et libre administration des collectivités territoriales », AJDA 2010, n² 28, p. 1595. 
n'atteint pas les chiffres nécessaires ${ }^{51}$, aussi le préfet refuse-t-il la fusion. Les communes maintiennent leur projet et adressent au préfet une nouvelle demande qu'il rejette. Le contentieux qui s'en suit amène les communes jusque devant le Conseil d'État ${ }^{52}$, lequel saisit le Conseil constitutionnel d'une question prioritaire de constitutionnalité, aux fins de savoir si la décision de procéder à une fusion de communes constitue ou non une atteinte au principe de libre administration des collectivités territoriales. La procédure prévue par le code général des collectivités territoriales, même si les critères de validation du référendum semblent restrictifs, n'en demeure pas moins conforme à la Constitution ${ }^{53}$, c'està-dire à l'article 72-1 relatif au principe de libre administration des collectivités.

Dans un second temps, à l'occasion de la décision Commune de Besançon $^{54}$, le Conseil constitutionnel reconnaît explicitement la possibilité de poser une question prioritaire de constitutionnalité sur le fondement du principe de la libre administration des collectivités territoriales. En l'espèce, le juge constitutionnel s'était assuré que l'accroissement des charges liées à la délivrance de cartes d'identités et de passeports, pesant sur les communes, ne présentait pas un poids tel à supporter par les communes que le principe de la libre administration des collectivités territoriales s'en serait trouvé dénaturé ${ }^{55}$. Le contentieux trouve son origine dans le transfert au profit des communes des dépenses liées à la réception et à la saisie des demandes de cartes nationales d'identité et de passeports. Le Conseil d'État avait déjà jugé auparavant, dans sa décision Commune de Versailles ${ }^{56}$, que les compétences confiées aux maires en matière de titres d'identité l'étaient en leur qualité d'agents de l'État ${ }^{57}$. La commune de Versailles, faisant état d'un surplus de dépenses, avait alors obtenu du juge administratif l'annulation du décret confiant aux communes la réception et la transmission des demandes de cartes d'identité ou de passeports. De nombreuses communes avaient dès lors adressé au Conseil d'État des demandes de remboursement pour les sommes versées. Dans la décision Commune de Besançon, le Conseil

51. Le projet avait recueilli la majorité requise, mais cette majorité ne correspondait pas au seuil du quart des électeurs inscrits sur les listes électorales.

52. CE, 18 mai 2010, $\mathrm{n}^{0}$ 306643, Commune de Dunkerque.

53. Cons. const., déc. 2 juil. 2010, $\mathrm{n}^{\mathrm{o}}$ 2010-12 QPC, Commune de Dunkerque.

54. Cons. const., déc. 22 sept. 2010, $\mathrm{n}^{\circ}$ 2010-29/37 QPC, Commune de Besançon.

55. Armelle Trepoz Bruant, "Libre administration des collectivités territoriales et QPC...", art. cité, p. 12.

56. CE, $\mathrm{n}^{\circ} 232888,5$ janv. 2005, Commune de Versailles.

57. Ibid., cons. 3 . 
d'État saisit le Conseil constitutionnel d'une question prioritaire de constitutionnalité en la matière. Si le juge constitutionnel reconnaît que le transfert des compétences en matière de titres d'identité a eu pour conséquence d'augmenter les dépenses des communes, en revanche il estime que cet accroissement n'est pas tel qu'il puisse être jugé disproportionné et, partant, ne constitue pas une atteinte au principe de libre administration des collectivités territoriales.

Ainsi, le mécanisme de la question prioritaire de constitutionnalité présente un nouveau souffle en matière de protection de la libre administration des collectivités territoriales. Le Conseil d'État, en s'en remettant au Conseil constitutionnel sur certains points, peut désormais affiner la définition et le contenu du principe de libre administration des collectivités. Lors de sa décision Syndicat mixte chargé de la gestion du contrat urbain de cohésion sociale de l'agglomération de Papeete ${ }^{58}$, le Conseil d'État choisit de surseoir à statuer pour poser la question au Conseil constitutionnel de la légalité des actes des communes de la Polynésie française. Le juge constitutionnel énonce à cette occasion ${ }^{59}$ «les conditions que doit respecter le contrôle administratif pour ne pas porter atteinte à la libre administration ${ }^{60} »$. Dans sa décision de renvoi Département des Landes ${ }^{61}$, le Conseil d'État est confronté à une interdiction législative faite aux départements de moduler les aides allouées aux communes en fonction du mode de gestion du service d'eau potable et d'assainissement qu'elles utilisent. Le Conseil constitutionnel, en réponse, pose que la libre administration intéresse les rapports entre les collectivités. Cette libre administration se compose donc d'une part de la libre disposition des ressources du département et d'autre part du libre choix du mode de gestion des services publics de la commune ${ }^{62}$. Et le Conseil constitutionnel juge que l'interdiction litigieuse constitue une restriction à la libre administration des départements qui est trop restrictive pour être conforme à la Constitution ${ }^{63}$, sans pour autant considérer

58. CE, $\mathrm{n}^{0} 343800,17$ déc. 2010, Syndicat mixte chargé de la gestion du contrat urbain de cohésion sociale de l'agglomération de Papeete.

59. Cons. const., déc. 17 mars 2011, $\mathrm{n}^{0}$ 2010-107 QPC, Syndicat mixte chargé de la gestion du contrat urbain de cohésion sociale de l'agglomération de Papeete [contrôle de légalité des actes des communes de la Polynésie française].

60. Armelle Trepoz Bruant, "Libre administration des collectivités territoriales et QPC... », art. cité, p. 13.

61. CE, $\mathrm{n}^{\circ} 347071,29$ avr. 2011, Département des Landes.

62. Armelle Trepoz Bruant, "Libre administration des collectivités territoriales et QPC...", art. cité, p. 13.

63. Cons. const., déc. 8 juil. 2011, $\mathrm{n}^{0}$ 2011-146 QPC, Département des Landes [aides publiques en matière d'eau potable ou d'assainissement]. 
que cette prévalence accordée à la libre administration des départements sur celle des communes constitue une tutelle d'une collectivité territoriale sur l'autre. Enfin, à l'occasion de la décision Départements de la Seine-Saint-Denis et $d u \operatorname{Var}^{64}$, le Conseil d'État s'intéresse à la relation entre le principe de libre administration des collectivités territoriales et le mécanisme de péréquation, et renvoie la question au Conseil constitutionnel, lequel répond en affirmant la compatibilité de la péréquation avec la libre administration, dans la mesure où les collectivités disposent de ressources suffisantes ${ }^{65}$.

Il ressort de cette jurisprudence administrative, complétée par la jurisprudence constitutionnelle, que le contrôle des actes des collectivités territoriales tend à devenir plus effectif et que la protection de leur libre administration en est renforcée. Certes, les décisions n'aboutissent pas toujours dans le sens espéré par les collectivités, mais il n'en demeure pas moins que lorsque les restrictions au principe de libre administration sont manifestes, elles font en outre l'objet d'un contrôle de proportionnalité afin de déterminer si elles peuvent être ou non supportées par la collectivité. Dès lors, l'introduction du contrôle des actes des collectivités territoriales pris en vertu de leur droit de s'administrer librement par la QPC permet d'enrichir le contenu du principe de libre administration et, à défaut d'obtenir une définition tranchée, de mieux en saisir les contours.

Une fois esquissée une définition de la libre administration des collectivités territoriales et constatée la réalité du contrôle des actes pris par les collectivités, la question se pose de savoir comment cette libre administration s'exerce concrètement. Ainsi, si la protection du principe de libre administration des collectivités est assurée par une reconnaissance d'une part, et un double contrôle d'autre part, aussi bien constitutionnels qu'administratifs, il importe néanmoins de rechercher dans quelle mesure ce principe et sa protection sont rendus effectifs.

\section{L'effectivité du principe de libre administration des collectivités territoriales}

De la même manière qu'il a posé la règle selon laquelle il appartient au pouvoir réglementaire de répartir les compétences entre les agents

64. CE, $\mathrm{n}^{\circ}$ 356633, 21 mai 2012, Départements de la Seine-Saint-Denis et du Var.

65. Cons. const., déc. 29 juin 2012, $\mathrm{n}^{0}$ 2012-255/265 QPC, Départements de la SeineSaint-Denis et $d u$ Var [fonds national de péréquation des droits de mutation à titre onéreux perçus par les départements]. 
de l'État ${ }^{66}$, le Conseil d'État décide que seul le législateur est compétent pour imposer une dépense aux communes ${ }^{67}$. Il arrive que les compétences attribuées à l'État soient transférées aux collectivités territoriales, entraînant de ce fait " une charge supplémentaire pour les services ${ }^{68}$ " d'une commune ou d'un département. Le juge administratif connaît régulièrement du contentieux entourant le transfert des compétences de l'État vers une collectivité, ou d'une collectivité envers une autre ou encore de la mise en compatibilité des documents d'urbanisme, en particulier lorsque de nouveaux documents sont imposés par l'État aux collectivités (2.1). En outre, se pose la question de l'autonomie financière des collectivités territoriales, en tant que corolaire de leur principe de libre administration (2.2).

\subsection{Le partage des compétences entre l'État et les collectivités territoriales}

L'article 72 de la Constitution confère aux collectivités territoriales le droit de s'administrer librement. Toutefois, rien n'empêche le gouvernement d'intervenir de façon complémentaire dans le domaine de l'administration locale. En effet, l'article 21 de la Constitution prévoit que le Premier ministre dispose du pouvoir réglementaire. C'est la raison pour laquelle d'aucuns se demandent si cette habilitation qui se passe de l'intervention du législateur pour être valide, n'aboutit pas à « effacer toute habilitation légale au profit des collectivités ${ }^{69}$ ». Le Conseil d'État s'attache à répondre à la question de l'articulation du pouvoir réglementaire du Premier ministre et celui des collectivités territoriales, de façon synthétique, dans son avis rendu le 15 novembre $2012^{70}$. Il s'appuie sur sa propre jurisprudence pour affirmer que le Premier ministre, en vertu de l'article 34 de la Constitution, ne peut intervenir dans le domaine de la libre administration des collectivités territoriales que si le législateur lui en donne l'habilitation ${ }^{71}$. Il confirme ainsi sa décision rendue à l'occasion de l'arrêt Département de la Loire de $1997^{72}$. En l'espèce, le département de la Loire soutenait que le ministre de la Santé aurait dû

66. CE, n 215999, 27 juin 2001, Commune de Maison-Lafitte.

67. CE, $\mathrm{n}^{\mathrm{O}} 232888,5$ janv. 2005, Commune de Versailles, cons. 3.

68. Emmanuel Glaser, «Principe de libre administration des collectivités territoriales», Revue Lamy des collectivités territoriales, $\mathrm{n}^{0} 1$, avril 2005, p. 13.

69. Bertrand Faure, "Le Conseil d'État et le pouvoir réglementaire des collectivités territoriales», AJDA 2013, n ${ }^{\circ}$ 39, p. 2244.

70. CE, avis, 15 nov. 2012, $\mathrm{n}^{\circ} 387.095$, Pouvoir réglementaire.

71. Ibid., point 4 a.

72. CE, nº 142266/142390, 19 mars 1997, Département de la Loire. 
contresigner le décret du 28 août 1992 portant statut particulier du cadre d'emplois des conseillers territoriaux socio-éducatifs, ce qu'il n'avait pas fait. Le juge administratif répond que le ministre de la Santé n'était pas " chargé de l'exécution du décret attaqué ${ }^{73}$ » et qu'il ne lui revenait nullement de contresigner ledit décret. Par cette décision, le Conseil d'État marque bien l'autonomie des compétences des collectivités par rapport à celle du pouvoir exécutif, et rappelle qu'il n'y a pas d'interdépendance d'office entre les deux.

Toutefois, cela ne revient pas à dire que l'État et les collectivités agissent parallèlement. Le Conseil d'État insiste sur l'ordre dans lequel les règles doivent être édictées et sur le fait qu'un décret d'application "ne doit pas empiéter sur les compétences réglementaires que la loi a confiées aux collectivités territoriales ${ }^{74} »$. Ainsi, dans un autre contentieux concernant le département de la Loire ${ }^{75}$, le Conseil d'État prévoit clairement la répartition des tâches entre les collectivités et l'État. En l'espèce, il rappelle que la loi donne compétence aux départements pour " organiser et gérer les services de la protection maternelle et infantile», tandis que la Constitution réserve à l'État «le soin de fixer les normes minimales d'activité de ces services ${ }^{76}$ ", c'est-à-dire les normes relatives à l'encadrement des activités et aux exigences de qualification des personnes employées. Dans l'avis Préfet du Calvados ${ }^{77}$, le conseil rappelle que l'édiction des règles confiées par la loi aux collectivités territoriales ne doit pas intervenir avant la mise en place du cadre général dont la détermination est renvoyée par décret.

Malgré cela, le Conseil d'État reconnaît qu'il n'y a pas de franche étanchéité entre les règles confiées au décret et celles relevant des collectivités territoriales ${ }^{78}$. Il prévoit dès lors que l'abstention partielle du pouvoir réglementaire national laisse la place au pouvoir réglementaire local ${ }^{79}$ dans une décision concernant les opérations électorales pour la représentation des personnels sapeurs-pompiers à la commission administrative du service départemental d'incendie et de secours. Le Conseil d'État ajoute qu'un décret ne peut, de sa propre initiative, confier aux collectivités le soin de fixer ses modalités d'application, une habilitation législative étant nécessaire, sans quoi il y aurait atteinte au principe de

73. Ibid., cons. 2.

74. CE, avis, 15 nov. 2012, $\mathrm{n}^{\mathrm{0}} 387.095$, Pouvoir réglementaire, point $4 \mathrm{~b}$.

75. CE, $\mathrm{n}^{\mathrm{o}} 141958,1^{\mathrm{er}}$ avr. 1996, Département de la Loire.

76. Ibid. cons. 2.

77. CE, avis, 20 mars 1992, $\mathrm{n}^{\circ}$ 131852, Préfet du Calvados.

78. CE, avis, 15 nov. 2012, $\mathrm{n}^{\mathrm{0}} 387.095$, Pouvoir réglementaire, point $4 \mathrm{c}$.

79. CE, $\mathrm{n}^{\circ}$ 90306, 10 juin 1998, Département de l'Orne. 
libre administration des collectivités territoriales ${ }^{80}$. Enfin, dans l'arrêt Syndicat communautaire d'aménagement de Cergy-Pontoise ${ }^{81}$, confirmé par l'arrêt Commune de Cuers ${ }^{82}$, le conseil précise qu'en cas d'absence de renvoi au décret par une loi relative aux compétences locales, il y a habilitation indirecte des collectivités territoriales à arrêter elles-mêmes les règles utiles à l'exercice de ces compétences.

Force est dès lors de constater que le constituant, aidé du législateur, entend faire collaborer l'État et ses collectivités en matière d'administration de ces dernières. En effet, le principe de libre administration des collectivités territoriales n'empêche nullement l'État d'intervenir, puisque celui-ci conserve "les rênes du pouvoir politique ${ }^{83}$ ». Cela s'observe notamment en matière de dissolution des conseils municipaux, à laquelle le pouvoir exécutif peut procéder, s'immisçant ainsi dans l'administration locale ${ }^{84}$. Toutefois, le Conseil d'État précise que le décret doit présenter de façon expresse les motifs ayant amené l'État à dissoudre l'instance communale ${ }^{85}$. Il accepte cependant que ces motifs soient présentés de façon laconique, comme dans l'arrêt Caltabellotta ${ }^{86}$. En l'espèce, le conseil municipal d'une commune de Bourgogne avait refusé d'approuver le budget communal. Le décret de dissolution concernant ce conseil expliquait simplement que «les dissensions qui existent au sein du conseil municipal de Brain entravent l'administration de cette commune $^{87}$ ", décret que n'annule pas le Conseil d'État. Mais si le pouvoir exécutif semble bénéficier de prérogatives primant celles des collectivités, dans un domaine qui pourtant leur revient, en contrepartie l'État assume seul la responsabilité des erreurs des collectivités s'agissant du droit communautaire. En effet, «l'État est le seul garant du droit communautaire sur son territoire ${ }^{88} "$, si bien qu'alors même qu'un

80. CE, avis, 15 nov. 2012, $\mathrm{n}^{\mathrm{0}} 387.095$, Pouvoir réglementaire, point $4 \mathrm{~d}$.

81. CE, $\mathrm{n}^{\circ} 40756,13$ févr. 1985, Syndicat communautaire d'aménagement de l'agglomération nouvelle de Cergy-Pontoise.

82. CE, $\mathrm{n}^{\circ}$ 148121, 2 déc. 1994, Commune de Cuers.

83. Olivier Carton, "La dissolution de conseils municipaux par décret en Conseil des ministres : une prérogative étatique discrétionnaire? ", Revue Lamy des collectivités territoriales, $\mathrm{n}^{\circ} 68$, mai 2011, p. 44.

84. CE, $\mathrm{n}^{\mathrm{o}} 339145,23$ mars 2011, Becavin et autres.

85. CE, no 93824, 19 janv. 1990, Bodin.

86. CE, $\mathrm{n}^{\circ}$ 295296, 4 juin 2007, René Caltabellotta.

87. Olivier Carton, «La dissolution de conseils municipaux par décret en Conseil des ministres...», art. cité, p. 45.

88. Catherine Gauthier, «Comment concilier la responsabilité exclusive de l'État en droit communautaire avec, en droit interne, les principes de libre administration des 
manquement est imputable à une collectivité territoriale, l'État demeure responsable à sa place ${ }^{89}$.

Dans le domaine des documents d'urbanisme, la question de la répartition et de la complémentarité des compétences se pose à travers le problème de la mise en compatibilité de tels documents. En effet, se trouve d'un côté l'élaboration d'un document d'urbanisme qui relève du pouvoir discrétionnaire de la commune, puisque cet acte est rattaché à la libre administration des collectivités territoriales ${ }^{90}$. De l'autre côté se trouve la modification, par le biais de la mise en compatibilité, de ce document dont la compétence appartient à l'État. L'arrêt Thalineau ${ }^{91}$ du Conseil d'État met en lumière la position de ce dernier sur ce point. En l'espèce, un décret de 2009 déclarant d'utilité publique les travaux nécessaires à la réalisation d'un tronçon de ligne ferroviaire et emportant mise en compatibilité des documents d'urbanisme d'un certain nombre de communes est attaqué par $\mathrm{M}$. Thalineau. Le requérant demande que soit posée au Conseil constitutionnel une question prioritaire de constitutionnalité aux fins de savoir si cette mise en compatibilité n'entrave pas la libre administration des communes concernées. Le Conseil d'État choisit de ne pas soumettre la question au Conseil constitutionnel, estimant non rempli le critère du caractère sérieux de la question ${ }^{92}$. Le juge administratif estime en effet que, bien que la mise en compatibilité d'office d'un document d'urbanisme porte atteinte au principe de libre administration des collectivités territoriales, puisque ne tenant pas compte de l'avis de la commune, le législateur est néanmoins habilité à limiter ce principe constitutionnel dans un but d'intérêt général ${ }^{93}$. Il s'appuie pour cela sur la jurisprudence en la matière du Conseil constitutionnel, notamment la décision Quartier d'affaires de La Défense de $2007^{94}$. Ce faisant, le Conseil d'État s'inscrit

collectivités territoriales et d'organisation décentralisée de la République? », JCP A 2004, $\mathrm{n}^{\mathrm{o}} 38$, p. 1577.

89. Edwige Belliard et Catherine de Salins, Collectivités territoriales et obligations communautaires. Étude adoptée le 23 octobre 2003 par le Conseil d'État, Paris, La Documentation française, 2004, $114 \mathrm{p}$.

90. Seydou Traoré, «La mise en compatibilité d'un document d'urbanisme par une DUP n'est pas contraire à la libre administration des collectivités territoriales", Revue Lamy des collectivités territoriales, $\mathrm{n}^{\circ} 64$, janvier 2011, p. 41.

91. CE, $\mathrm{n}^{0} 330734,15$ sept. 2010, M. Thalineau.

92. Ibid., cons. 6 .

93. Jérôme Tremeau, «La mise en compatibilité des documents d'urbanisme avec une opération déclarée d'utilité publique est-elle contraire au principe de libre administration des collectivités territoriales? ", BJDU 2010, nº 6, p. 436.

94. Cons. const., déc. 22 fév. 2007, $\mathrm{n}^{\circ}$ 2007-548 DC, Quartier d'affaires de la Défense. 
dans le droit fil de sa propre jurisprudence, dégagée à l'occasion de l'arrêt Commune de Proville ${ }^{95}$, dans lequel le juge administratif avait admis que "la théorie des mutations domaniales ne méconnaissait pas le principe de la libre administration des collectivités territoriales, consacrant ainsi la primauté accordée à l'intérêt général sur les intérêts publics particuliers $^{96}$ ».

De la même manière, la procédure de projet d'intérêt général (PIG) permet également à l'État d'imposer aux communes d'intégrer dans les documents d'urbanisme locaux des projets dont l'enjeu dépasse le cadre communal ${ }^{97}$, faisant primer l'intérêt général. L'atteinte au principe de libre administration des collectivités territoriales est donc manifeste. Dans son arrêt Parc d'activité de Blotzheim et autres ${ }^{98}$, le Conseil d'État décide de surseoir à statuer et de renvoyer au Conseil constitutionnel, en faisant jouer le mécanisme de la QPC, la question de savoir si cette atteinte n'est pas telle qu'elle aboutirait à dénaturer le principe de la libre administration des collectivités. Le Conseil constitutionnel répond que la définition de la nature du PIG ne met pas en cause les principes fondamentaux de la libre administration des collectivités territoriales ${ }^{99}$. L'intérêt général semble dès lors constituer le critère de référence permettant de déterminer si une atteinte portée au principe de libre administration des collectivités est acceptable ou non. L'atteinte se justifie au nom de l'intérêt général, motif grâce auquel une restriction à ce principe constitutionnel peut être considérée comme ne le dénaturant pas de telle manière que la restriction soit inconstitutionnelle.

La répartition des compétences telles que contrôlée par le Conseil d'État préserve la réalité du principe de libre administration des collectivités et lui assure une réelle protection. La question de l'effectivité de la libre administration des collectivités territoriales se pose en des termes spécifiques s'agissant de l'autonomie financière des collectivités, par la coexistence des compétences confiées aux collectivités et à l'État. Cette autonomie financière concerne les dépenses dues aux transferts de compétence, le recours au mécanisme de péréquation ou la manière dont

95. CE, $\mathrm{n}^{\mathrm{O}}$ 253419, 23 juin 2004, Commune de Proville.

96. Mattias Guyomar (rapporteur public), Conclusions présentés dans l'arrêt suivant: $\mathrm{CE}, \mathrm{n}^{\mathrm{o}} 330734,15$ sept. 2010, M. Thalineau.

97. Jérôme Tremeau, «L'ex-article L. 121-9 pouvait-il renvoyer au règlement la définition de la nature des projets d'intérêt général ? ", BJDU 2011, nº 3 , p. 188.

98. CE, $\mathrm{n}^{\circ} 340213,26$ nov. 2010, SARL du parc d'activités de Blotzheim et autres.

99. Cons. const., déc. 28 janv. 2011, $\mathrm{n}^{0}$ 2010-95 QPC, SARL du parc d'activités de Blotzheim et autres. 
les collectivités entendent gérer le principe du non-subventionnement du culte.

\subsection{La question de l'autonomie financière}

La Constitution consacre en son article 72-2 le principe de l'autonomie financière des collectivités territoriales ${ }^{100}$, en leur assurant la liberté de disposer des ressources dont elles bénéficient. Ce principe est régulièrement raffermi ${ }^{101}$ et la question des finances locales a beaucoup évolué ces trente dernières années, aussi bien s'agissant du contrôle, de la fiscalité locale, que des dépenses locales et de l'endettement des collectivités territoriales ${ }^{102}$. La Constitution précise que les ressources des collectivités proviennent en tout ou partie des impositions de toutes natures, ainsi que des recettes fiscales propres aux collectivités ${ }^{103}$. Enfin, les collectivités territoriales perçoivent également des ressources de l'État, notamment en cas de transferts de compétences, lesquels doivent s'accompagner de "ressources équivalentes à celles qui étaient consacrées à leur exercice ${ }^{104}$ ». Pourtant, une partie des élus locaux estiment insuffisantes les ressources dont leurs collectivités disposent, regrettant que «l'État ne leur donne pas les moyens d'exercer les responsabilités que la loi leur confère ${ }^{105} »$. Le Conseil d'État est dès lors confronté à la question de savoir si la libre administration est réellement un « outil de protection efficace des collectivités ${ }^{106} »$.

Ainsi, dans sa décision d'avril 2011, Départements de la Seine-SaintDenis et de l'Hérault ${ }^{107}$, le Conseil d'État accepte de poser au Conseil constitutionnel une question prioritaire de constitutionnalité s'agissant d'un transfert de compétences en matière de paiement de l'allocation

100. «Les collectivités territoriales bénéficient de ressources dont elles peuvent disposer librement dans les conditions fixées par la loi» (art. 72-2 al. 1 de la Constitution du 4 octobre 1958).

101. Marie-Christine Steckel Assouère, «Les EPCI à fiscalité propre, fossoyeurs des communes?», BJCL 2013, $n^{\circ}$ 10, p. 652.

102. Xavier Cabannes, "Trente ans après ou à la recherche de l'autonomie financière des collectivités territoriales", BJCL 2012, $\mathrm{n}^{\mathrm{O}}$ 4, p. 266.

103. Art. 72-2, al. 2 et 3 de la Constitution du 4 octobre 1958.

104. Art. 72-2 al. 4 de la Constitution du 4 octobre 1958.

105. Éric Giuily, Il y a 30 ans, l'Acte I de la décentralisation ou l'histoire d'une révolution tranquille, Paris, Berger-Levrault, 2012, p. 188.

106. Sylvie Joubert, «Les départements en difficulté financière, l'ultime épreuve pour le principe de libre administration ", Revue Lamy des collectivités territoriales, $\mathrm{n}^{\circ} 70$, juillet-août 2011, p. 14.

107. CE, $\mathrm{n}^{0}$ 346204, 20 avr. 2011, Départements de la Seine-Saint-Denis et de l'Hérault et autres. 
de revenu minimum d'insertion (ci-après, RMI/RSA). Le versement de cette allocation relève désormais des départements, mais l'augmentation du montant du RSA ne s'est pour autant pas accompagnée d'une augmentation des ressources des collectivités. En l'espèce, certains départements se plaignent d'une surcharge financière qu'ils ne peuvent plus assumer, liée à la diminution de leurs ressources doublée de l'augmentation des prestations sociales, tant s'agissant de leur montant que du nombre de personnes pouvant y prétendre. Le Conseil d'État reconnait que ce changement de circonstances rend la question nouvelle et permet qu'elle soit transmise au Conseil constitutionnel. Ce dernier conclut que la législation litigieuse demeure conforme à l'article 72 de la Constitution ${ }^{108}$. En matière de transfert de compétences, le Conseil d'État s'est également prononcé sur le dispositif de compensation au profit des régions s'agissant des transports collectifs d'intérêt régional, en jugeant que le transfert de compétences dans ce domaine ne devait pas avoir pour effet d'entraver la libre administration des régions ${ }^{109}$. Quant aux contentieux réclamant une QPC, le juge administratif ne répond pas systématiquement de façon positive. En effet, il accepte de transmettre la question concernant l'absence de compensation prévue par la loi dans le domaine de la protection juridique des majeurs dont la compétence revient désormais aux départements, jugeant du caractère sérieux de la question ${ }^{110}$. En revanche, il n'estime pas sérieux le caractère de la question relative au transfert de compétences au profit des départements des routes nationales, parce qu'en l'espèce le législateur n'avait pas envisagé les dépenses futures liées à ce transfert ${ }^{111}$. Les dépenses actuelles sont prises en compte par le législateur, mais pas les dépenses futures, celles-ci pouvant n'être qu'éventuelles. Enfin, le Conseil d'État a admis le caractère sérieux de la question relative à la réforme sur la protection de l'enfance ${ }^{112}$, estimant que la loi n'avait pas «suffisamment précisé les conditions et garanties de la compensation prescrite par la constitution ${ }^{113} »$. Le critère que le Conseil d'État semble retenir pour juger du caractère nouveau ou sérieux d'une question à transmettre au Conseil constitutionnel semble être celui de l'entrave faite

108. Cons. const., déc. 30 juin 2011, $\mathrm{n}^{\circ}$ 2011-143 QPC, Départements de la-Seine-SaintDenis et de l'Hérault [concours de l'État au financement par les départements de l'allocation personnalisée autonome], cons. 14 et 15 .

109. $\mathrm{CE}, \mathrm{n}^{\mathrm{o}} 339842,25$ juin 2010, Région Lorraine.

110. CE, $\mathrm{n}^{\circ} 340028,19$ juil. 2010, Département du Val-de-Marne.

111. CE, $\mathrm{n}^{\mathrm{o}} 342072,29$ oct. 2010, Département de la Haute-Garonne.

112. CE, $\mathrm{n}^{\mathrm{o}} 341612$, 30 déc. 2010, Département des Côtes-d'Armor.

113. Sylvie Joubert, «Les départements en difficulté financière...», art. cité, p. 17. 
à la libre administration des collectivités territoriales. Si le financement qui accompagne le transfert de compétences n'alourdit pas les charges pesant déjà sur la collectivité ou si un déséquilibre n'est qu'envisagé pour l'avenir, le Conseil d'État ne juge pas qu'il s'agisse d'un caractère suffisamment sérieux pour justifier un recours au mécanisme de la question prioritaire de constitutionnalité.

Le principe de péréquation, prévu à l'article 72-2 de la Constitution ${ }^{114}$, ne peut quant à lui être invoqué à l'appui d'une question prioritaire de constitutionnalité ${ }^{115}$. La péréquation est un mécanisme de redistribution visant à réduire les écarts de richesse entre les collectivités territoriales, diminuant ce faisant les risques d'inégalité entre elles. Il existe la péréquation verticale, c'est-à-dire de l'État vers les collectivités, et la péréquation horizontale, c'est-à-dire entre les collectivités territoriales. La péréquation verticale est assurée par les dotations de l'État, la péréquation horizontale résulte d'une redistribution des ressources entre les collectivités, les collectivités les plus pauvres recevant une partie des ressources des collectivités les plus riches. La péréquation permet à la fois de corriger les « inégalités affectant les ressources mais également les inégalités relatives aux charges ${ }^{116}$ ». Le mécanisme de la péréquation ne doit toutefois pas aboutir à un prélèvement trop lourd, pouvant générer une atteinte au principe de libre administration des collectivités territoriales ${ }^{117}$. Le Conseil d'État a eu à connaître du contentieux entourant le principe de péréquation à l'occasion de la décision Commune de Vitry-sur-Seine de $2012^{118}$. En l'espèce, le conseil juge que le fait qu'une commune éligible à la dotation de solidarité urbaine et de cohésion sociale puisse être contributrice au fonds national de péréquation des ressources intercommunales et communales, ne constitue pas une atteinte au principe de libre administration des collectivités territoriales ${ }^{119}$. À première vue, il aurait été plausible de penser qu'une collectivité qui bénéficie d'une dotation spéciale destinées

114. Art. 72-2 al. 4 de la Constitution du 4 octobre 1958: «La loi prévoit des dispositifs de péréquation destinés à favoriser l'égalité entre les collectivités territoriales ».

115. Cons. const., déc. 22 sept. 2010, $\mathrm{n}^{\circ}$ 2010-29/37 QPC, Commune de Besançon, cons. 5 .

116. "Observations sur CE, décision QPC du 21 septembre 2012, Commune de Vitrysur-Seine, req. $\mathrm{n}^{\circ} 360602$ ", RJF 2012, $\mathrm{n}^{\circ}$ 1151, p. 1028.

117. Vincent Daumas, "Le mécanisme de péréquation nationale des ressources intercommunales et communales est-il conforme à la Constitution?", BDCF 2012, $\mathrm{n}^{\mathrm{o}} 12, \mathrm{p} .44$.

118. CE, $\mathrm{n}^{\mathrm{o}}$ 360602, déc. QPC du 21 sept. 2012, Commune de Vitry-sur-Seine.

119. Ibid., cons. 13 . 
aux collectivités «confrontées à une insuffisance de leurs ressources et supportant des charges élevées ${ }^{120}$ ", soit exonérée d'une quelconque contribution au fonctionnement du mécanisme de péréquation. Or, dans la mesure où la loi prévoit un plafonnement de cette contribution à $10 \%$ des ressources prises en compte pour le calcul du potentiel fiscal ${ }^{121}$, le Conseil d'État en vient à la conclusion que les ressources de collectivité en litige ne se trouvent pas restreintes « au point de dénaturer le principe de libre administration [des] collectivités ${ }^{122} »$. Il juge également que l'augmentation des dépenses générées ne porte pas atteinte au principe de libre administration des collectivités territoriales et décide de ne pas renvoyer la question au Conseil constitutionnel. Le Conseil d'État conclut de la même manière dans sa décision Commune de Chambéry ${ }^{123}$, dans laquelle il estime que la législation litigieuse ne diminue pas le montant des ressources propres des communes et, partant, ne porte pas atteinte au principe de libre administration des collectivités territoriales. En effet, pour lui, «le respect des principes de l'autonomie financière et de la libre administration implique l'absence de diminution ou une diminution suffisamment limitée pour ne pas entraver la libre administration des collectivités territoriales, de la part de leurs ressources propres $^{124} »$. En l'espèce, jugeant de l'absence de diminution de ces ressources, le Conseil d'État conclut à la non-violation du principe de libre administration.

Sur cette question de l'égalité entre les collectivités territoriales, notamment en matière financière, le Conseil d'État confirme son contrôle de proportionnalité de l'atteinte faite au principe de libre administration des collectivités territoriales. En effet, dans sa décision Société « Au verger de Provence ${ }^{125}$ », le juge administratif opère une conciliation entre le principe d'égalité devant les charges publiques et le principe de libre administration des collectivités territoriales ${ }^{126}$. Le conseil estime que ce dernier principe n'impose pas au législateur de prévoir une règle uniforme sur l'ensemble du territoire national et que la libre administration

120. Art. L. 2334-15 du code général des collectivités territoriales.

121. «Observations sur CE, décision QPC du 21 septembre 2012, Commune de Vitrysur-Seine, req. $\mathrm{n}^{\circ} 360602$ ", RJF 2012, $\mathrm{n}^{\circ} 1151$, p. 1028.

122. CE, $\mathrm{n}^{0} 360602$, déc. QPC du 21 sept. 2012, Commune de Vitry-sur-Seine, cons. 12.

123. CE, $\mathrm{n}^{\circ} 331350,19$ juil. 2011, Commune de Chambéry.

124. Aurélie Duffy-Meunier, Laetitia Janicot et Agnès Roblot-Troizier, " Pouvoirs publics et autorités administratives ", NCCC 2012, $\mathrm{n}^{\circ} 36$, p. 178.

125. $\mathrm{CE}, \mathrm{n}^{\circ}$ 349382, 28 juil. 2011, Société "Au verger de Provence».

126. Aurélie Duffy-Meunier, Laetitia Janicot et Agnès Roblot-Troizier, «Pouvoirs publics et autorités administratives ", art. cité, p. 174. 
des collectivités n'est pas entravée, ni le principe d'égalité bafoué, en cas de répartition différente des contributions sur le territoire ${ }^{127}$. Il n'y a pas, en l'espèce, selon lui, de rupture d'égalité devant les charges publiques, le principe d'égalité n'interdisant pas au législateur de traiter différemment les communes d'un même département ${ }^{128}$.

Le principe de l'autonomie financière des collectivités territoriales se traduit également par une vision particulière de la règle de nonsubventionnement des cultes. En effet, confronté à la question de la légalité des dépenses des collectivités territoriales en rapport avec des édifices du culte, le Conseil d'État a précisé la portée et les limites de l'interdiction de subventionner les cultes et a aménagé des possibilités pour les collectivités de financer des équipements ou activités liés aux cultes ${ }^{129}$. La loi de 1905 dispose en son article 2 que «la République ne reconnait, ne salarie ni ne subventionne aucun culte ${ }^{130} »$. Si les associations cultuelles ne posent pas de difficultés particulières par rapport à cette législation, en revanche un problème surgit s'agissant des associations mixtes, proposant à la fois des activités liées au culte et des activités éducatives, sociales ou encore sportives. La question se pose en effet de savoir si ces associations peuvent bénéficier d'une aide de l'État et, plus précisément, des collectivités territoriales. La jurisprudence du Conseil d'État a évolué quant à la réponse apportée à cette question. Dans un premier temps, dans la décision Commune de Saint-Louis de la Réunion ${ }^{131}$, le juge administratif estime que la loi de 1905 s'étend aux associations présentant des activités mixtes et leur refuse ainsi tout subventionnement. Le conseil a longtemps fait valoir cette règle du nonsubventionnement des cultes pour préciser qu'une commune ne pouvait pas apporter de soutien financier même indirect à un culte, par exemple en louant à très bas prix un presbytère à un ministre du culte ${ }^{132}$. Il a également rappelé l'interdiction pour un ministre du culte de percevoir

127. CE, $n^{0}$ 349382, 28 juil. 2011, Société «Au verger de Provence», cons. 4.

128. "Observations sur CE, décision QPC du 21 septembre 2012, Commune de Vitrysur-Seine, req. ${ }^{\circ} 360602$ ", RJF 2012, $\mathrm{n}^{\circ} 1151$, p. 1028.

129. Jean-François Amédro, «Les collectivités territoriales et les cultes : le Conseil d’État précise la portée et les limites de la règle de non-subventionnement de l'exercice du culte», JCP A 2011, n 39, p. 2308.

130. Alinéa 1 de l'article 2 de la loi du 9 décembre 1905 concernant la séparation des églises et de l'État.

131. CE, $\mathrm{n}^{\circ}$ 94455, 9 oct. 1992, Commune de Saint-Louis de la Réunion contre association Shiva Soupramanien de Saint-Louis.

132. Voir notamment, CE, 16 déc. 1910, Commune de Callian, Recueil Lebon, 1910, p. 976 ; CE, 22 avr. 1910, Commune de Labastide-Saint-Pierre, Recueil Lebon, 1910, p. 329 et CE, 12 mars 1909, Commune de Triconville, Recueil Lebon, 1909, p. 275. 
une aide financière lui permettant d'accomplir les actes religieux liés à son ministère ${ }^{133}$. De la même manière, une commune n'est pas autorisée à attribuer à un séminariste une bourse dans le but de lui permettre de financer ses études liées à la préparation à la prêtrise ${ }^{134}$, ni à financer l'organisation d'une messe célébrée par le pape à l'occasion de sa venue dans la ville ${ }^{135}$. La décision Commune de Saint-Louis de la Réunion ne faisait en définitive qu'entériner une jurisprudence constante du juge administratif.

En 2011, le Conseil d'État revient implicitement sur cette jurisprudence ${ }^{136}$ en décidant qu'une "collectivité publique peut accorder une subvention destinée à la réalisation d'un équipement en rapport avec un édifice du culte, pourvu qu'il soit garanti [...] que la subvention n'est pas versée à une association cultuelle, et qu'elle est exclusivement affectée au financement de l'équipement ${ }^{137}{ }^{\prime}$. Cette décision laisse entendre que, dès lors qu'une association n'est pas uniquement cultuelle et que l'activité financée ne concerne pas le culte, un subventionnement est possible. Toutefois, outre l'exigence de la réserve exclusive du financement pour une activité non cultuelle, le Conseil d'État ajoute celle de la justification du subventionnement par un intérêt public local ${ }^{138}$. La difficulté réside cependant dans le fait que la plupart des associations mixtes ne présentent pas de nette distinction dans leurs activités entre celles relevant du cultuel pur et celles relevant exclusivement de l'éducatif ou du social. En tout état de cause, la décision du 4 mai $2012^{139}$ vient confirmer le revirement de jurisprudence opéré par rapport à l'arrêt de 1992, en posant que «la loi de 1905 ne fait pas obstacle, sous certaines conditions, à ce que les personnes publiques puissent accorder une subvention à des associations mixtes ayant des activités cultuelles et non cultuelles ${ }^{140}$ ».

133. CE, 21 mai 1909, Commune de Saint-Michel de Volangis et abbé Garcin et autres, Recueil Lebon, 1909, p. 886.

134. CE, 13 mars 1953, Ville de Saumur, Recueil Lebon, 1953, p. 130.

135. TA Châlons-en-Champagne, 18 juin 1996, Thierry Come, association Agir contre Ville de Reims (RFDA 1996, p. 1012, conclusion Daniel Warin).

136. CE, $\mathrm{n}^{0} 308817,19$ juil. 2011, Fédération de la libre pensée et de l'action sociale du Rhône.

137. Jean-François Amédro, «Le Conseil d'État poursuit son effort de redéfinition des contours de la règle de non-subventionnement des cultes: l'admission sous conditions du financement public des activités non cultuelles des associations mixtes », JCP A 2012, $n^{0}$ 27, p. 27.

138. Ibid., p. 28.

139. CE, $\mathrm{n}^{\mathrm{o}} 336462,4$ mai 2012, Fédération de la libre pensée et de l'action sociale du Rhône.

140. Jean-François Amédro, «Le Conseil d'État poursuit son effort de redéfinition des contours de la règle de non-subventionnement des cultes...», art. cité, p. 26. 
Le Conseil permet ainsi explicitement aux collectivités territoriales de financer certains projets des associations mixtes, toujours dans le respect des conditions énoncées en 2011. Cette décision de 2012 parachève une jurisprudence administrative plus souple à l'égard du subventionnement des activités non cultuelles mais rattachées à un culte. Par exemple, la décision Commune de Trélazé $e^{141}$ amène le Conseil d'État à permettre l'acquisition par une commune d'un orgue, en vue de le restaurer et de l'installer dans l'église dont la commune est propriétaire, dans le but de favoriser le déploiement des politiques locales de valorisation des églises ${ }^{142}$. L'arrêt Communauté urbaine du Mans ${ }^{143}$ intervient en matière d'abattage rituel et permet à une collectivité territoriale d'aménager, dans le cadre des fêtes juives et musulmanes, un local en abattoir afin de le mettre à disposition d'un culte. Le Conseil d'État se montre favorable à cette mise à disposition, dans la mesure où cela permet un contrôle des mesures d'hygiène et du respect des exigences de la santé publique, d'une part, et d'autre part, sous réserve de louer l'abattoir et non pas de le mettre gratuitement à disposition d'une association cultuelle. De la même manière, le Conseil autorise la location à une association mixte d'une salle communale polyvalente, le fait que l'association s'y réunisse pour y prier n'étant pas exclusif du prêt des locaux, à la condition qu'il y ait une contrepartie financière et que la prière soit une activité secondaire de l'association ${ }^{144}$. Les collectivités territoriales utilisent ainsi leur autonomie financière pour restaurer certaines églises et réactiver les activités touristiques de leur secteur, pour assurer le respect des droits fondamentaux tels que le droit à la santé. Le Conseil d'État autorise de plus en plus ces subventionnements du culte, toujours sous la réserve que l'aide apportée au culte ne soit pas à titre gratuit et demeure dans le respect du principe d'égalité ${ }^{145}$.

Le principe de libre administration des collectivités territoriales s'exerce concrètement à travers le mécanisme de compensation lors des transferts de compétences, le mécanisme de péréquation et le subventionnement sous condition des cultes. Le principe de l'autonomie financière contribue ainsi réellement à renforcer la libre administration des collectivités.

141. CE, $\mathrm{n}^{0}$ 308544, 19 juil. 2011, Commune de Trélazé.

142. Jean-François Amédro, "Les collectivités territoriales et les cultes...», art. cité, p. 2310.

143. CE, $\mathrm{n}^{0}$ 309161, 19 juil. 2011, Communauté urbaine du Mans - Le Mans Métropole.

144. CE, $\mathrm{n}^{\mathrm{o}}$ 313518, 19 juil. 2011, Commune de Montpellier.

145. Voir, notamment, CE, $\mathrm{n}^{\circ}$ 78589, 21 avr. 1972, Ville de Caen; CE, $\mathrm{n}^{\circ} 137376$, 15 mars 1996, Cavin; CE, n 304053, 30 mars 2007, Ville de Lyon. 
En conclusion, le principe de libre administration des collectivités territoriales bénéficie d'une protection incontestable. Certes, s'il jouit d'une assise constitutionnelle et d'une reconnaissance explicite du Conseil d'État, il ne présente pas encore de définition suffisante. Toutefois, cette définition encore trop hésitante ne constitue pas un frein à l'effectivité de la libre administration des collectivités. Le contrôle opéré par le préfet, renforcé par celui rendu possible avec la question prioritaire de constitutionnalité, enrichit le contenu du principe de libre administration des collectivités territoriales, en encadrant les actes par lesquels il se concrétise. Ce double contrôle renforce la protection de ce principe, même si l'atteinte n'est pas toujours reconnue. Le Conseil d'État se prononce sur les dépenses liées aux transferts de compétence et la mise en compatibilité des documents d'urbanisme, en s'assurant que les dépenses imposées par l'État ne grèvent pas tant le budget des collectivités que leur libre administration s'en trouverait paralysée. Enfin, le Conseil vérifie le respect du principe d'autonomie financière, composante du principe de libre administration, que les collectivités exercent notamment par le mécanisme de péréquation et leur choix de subventionner des associations cultuelles mixtes. Le principe de libre administration des collectivités territoriales semble trouver dans la jurisprudence du Conseil d'État une reconnaissance et une mise en œuvre concrète, aboutissant au renforcement de la protection de ce principe constitutionnel.

Docteur en droit public 